\title{
EDITORIAL
}

\section{The erythrocyte: a new player in atheromatous core formation}

\section{G Pasterkamp, R Virmani}

Heart 2002;88:115-116

The membrane of the red blood cell hides constituents that are lipid rich and can bind to macrophage scavenger receptors, thus posing the challenging hypothesis that erythrocytes contribute to atheroma formation in coronary arteries
See end of article for authors' affiliations

...........

Correspondence to: Dr G Pasterkamp, Experimental cardiology Laboratory, Utrecht Medical Center, Room G02-523, 3584 CX Utrecht, the Netherlands; g.pasterkamp@hli.azu.nl n atherosclerotic disease, proliferation of smooth muscle cells, matrix synthesis, and lipid accumulation narrows the lumen. Thrombus formation may be superimposed on mature plaques by rupture or superficial erosion of the atherosclerotic lesion ${ }^{1}$ leading to unstable angina and myocardial infarction. The three determinants of a plaque's vulnerability to rupture are the size of the atheromatous core, the thickness of the fibrous cap covering the core, and inflammation within the cap.

The size of the atheromatous core seems critical for the stability of the plaque. Postmortem studies revealed larger atheromatous cores in lesions that rupture compared with lesions that have an intact surface or in atheromas with a thin cap (vulnerable plaque). ${ }^{2}$ Furthermore, Davies and colleagues ${ }^{3}$ found a relation between core size and the occurrence of plaque rupture. They identified a threshold for rupture prone atherosclerotic plaques when the core occupied more than $40 \%$ of the atherosclerotic lesion. The consistency of the core also seems important for plaque stability. Liquid cholesteryl esters soften while crystalline cholesterol stiffens the atheroma. If temperature increases, as with inflammation, ${ }^{4}$ the core gets softer resulting in less stability. Virmani and colleagues reported a higher percentage of area occupied by the cholesterol cleft in sections with plaque rupture than in thin cap atheromas. ${ }^{2}$ The atheromatous core is rich in extracellular lipid, avascular, and the only cells are macrophages that are most frequently present at the edges of the core. It is generally accepted that macrophages take up oxidised low density lipoprotein (LDL) via scavenger receptors and become foam cells. These foam cells die by necrosis or apoptosis caused by the cytotoxic effects of internalised LDL, resulting in extracellular lipid accumulation.

\section{RED BLOOD CELLS AND ATHEROMA}

In the current issue, Arbustini and colleagues report on the composition of pulmonary artery plaques obtained from patients suffering from thromboembolic and plexogenic pulmonary hypertension. Their study provides a new cellular substrate for atheromatous core formation-the membrane of the erythrocyte. The atheroma rich lesions were mainly observed in chronic thromboembolic pulmonary hypertension and stained strongly positive for the erythrocyte specific membrane protein glycophorin.

About $40 \%$ of the weight of the erythrocyte is composed of lipid. The red cell membrane is comprised of a cholesterol rich phospholipid bilayer. Cholesterol is intercalated between the phospholipid molecules. The ratio of cholesterol and phospholipid content is related to the fluid properties of the erythrocyte membrane. Alterations in this ratio result in abnormal morphology and reduced life span. Glycophorins are sialoglycoproteins that span the lipid bilayer and are considered anion exchangers.

The results of the study by Arbustini and colleagues ${ }^{6}$ point to an origin of the large atheromatous core in pulmonary artery plaques as being something other than the lipid loaded macrophage. Although inflammatory cells were evident within the pulmonary artery lesions, the association between the atheromatous core and red cell membrane glycophorins is unexpected, has not been described previously, and therefore merits careful consideration. As suggested by the authors, extrapolation of these results to the coronary circulation would imply an amendment to the knowledge on the pathogenesis of the atheromatous rupture prone lesion. Although plaque composition in pulmonary hypertension may resemble plaques seen in systemic arteries, extrapolation of the results to the atherosclerosis prone arteries lacks a firm basis at present. Firstly, the aetiologies of pulmonary plexogenic arteriopathy and chronic thromboembolic pulmonary hypertension differ substantially from atherosclerotic lesion formation. Pulmonary hypertension in plexogenic disease arteriopathy (Eisenmenger) is caused by congenital heart disease or plexogenic pulmonary hypertension. Although still controversial, ${ }^{7}$ it is suggested that chronic thrombosis and embolism is a common cause of chronic pulmonary hypertension. The study by Arbustini and colleagues indeed confirms the differences in pathogenesis: the plaque phenotype in the pulmonary artery appeared independent of metabolic risk factors for atherosclerotic disease. Moreover, angiographically documented absence of coronary atherosclerosis was demonstrated in part of the affected population. Secondly, the lesions in thromboembolic pulmonary hypertension often revealed layered remnants of thrombi which raises the possibility that plaque accumulation is caused by fusion of pre-existing organised and new superficial thrombus formation. In atherosclerotic disease, organisation of intraplaque haemorrhage is considered a late stage event where smooth muscle cell migration 
and matrix formation are also major components of accumulating plaque. Finally, and most important, in the present study coronary arteries have not been studied for the presence of glycophorin in atheromatous core. This would have strengthened the intriguing hypothesis that in coronary arteries thrombotic events may give rise to the formation of thrombogenic atheroma.

\section{RED BLOOD CELLS: A SUBSTRATE FOR CORONARY ATHEROMA?}

Although aetiologies may differ for the diseases that predispose for pulmonary and systemic plaque formation, the substrate for atheroma (thrombus) has been described in both plaque entities. Plaque rupture and erosion may give rise to symptomatic and asymptomatic thrombus in the coronary circulation. Burke and colleagues ${ }^{8}$ demonstrated that multiple healed rupture sites with layering are frequently observed in men who died from sudden coronary death. In addition, carotid endarterectomy studies revealed that intraplaque haemorrhage is observed in the majority of specimens. ${ }^{9}$ Similarly plaque haemorrhage in coronary atherosclerotic plaques is a frequent phenomenon, especially in patients dying with plaque rupture rather than stable plaques. Thus, (organised) thrombus is a frequently observed feature in atherosclerotic plaques which theoretically makes the membrane of the red blood cell a contributor to atheroma formation in atherosclerosis prone arteries. Some of the free cholesterol present in the necrotic core may originate from the red cell membrane, which is 1.5-2.0 times richer in cholesterol than any other cell. An inherent limitation in research on atherogenesis is the lack of animal models and non-invasive techniques to study the process in vivo. The incidence of asymptomatic plaque rupture is unknown and histological studies often suffer from selection bias, which makes it difficult to estimate how often subclinical thrombus formation superimposed on atherosclerotic plaques occurs in the coronary circulation.

\section{THE MEMBRANE OF THE RED BLOOD CELL AND ATHEROGENESIS}

The erythrocyte is a cell with no nucleus and few organelles, and is considered a carrier of oxygen without a role in inflammatory responses. Cell surface components, however, can be recognised by macrophage scavenger receptors. The sialosaccharide chains of glycophorin A can act as ligands for macrophage scavenger receptors. This binding of glycophorin A seems competitive with the binding of oxidised LDL with the scavenger receptor. ${ }^{9}$ Thus, although speculative, when red blood cells undergo denaturation, like with oxidative stress, recognition of red cell membrane remnants by macrophages and subsequent internalisation of membrane originating lipids may provide another substrate for foam cell formation.

Another erythrocyte membrane constituent that has been associated with atherosclerosis is hexacosanoate (C26:0), which is a very long chain fatty acid (VLFA). Recently, Antoku and colleagues ${ }^{10}$ demonstrated an association with concentrations of erythrocyte membrane C26:0 and classical risk factors for atherosclerotic disease. Accumulation of C26:0 is suggested to result in a reduction of fluidity of membranes. An increase of C26:0 VLFA could crucially affect membrane functions in cell types, like the endothelial cell, that are thought to play pivotal roles in the initiation of atherosclerosis. It is noteworthy to point to a study of Singh and colleagues ${ }^{11}$ who found that lovastatin greatly reduces accumulation of VLFAs in adrenoleukodystrophy. Whether the relation between C26:0 and risk factors for atherosclerosis is causal remains to be investigated.

In summary, potentially the erythrocyte is a new player in atheromatous lesion formation. The red cell membrane hides constituents that are lipid rich, can bind to macrophage scavenger receptors, and are associated with risk factors for atherosclerotic disease. The hypothesis that red cell membranes contribute to atheroma formation in coronary arteries is challenging.

\section{Authors' affiliations}

G Pasterkamp, Experimental Cardiology Laboratory, Utrecht Medical Center and the Interuniversity Cardiology Institute of the Netherlands, Utrecht, The Netherlands

R Virmani, Department of Cardiovascular Pathology, Armed Forces Institute of Pathology, Washington DC, USA

\section{REFERENCES}

1 Farb A, Burke AP, Tang AL, et al. Coronary plaque erosion without rupture into a lipid core. A frequent cause of coronary thrombosis in sudden coronary death. Circulation 1996;93:1354-63.

2 Virmani R, Kolodgie FD, Burke AP, et al. Lessons from sudden coronary death: a comprehensive morphological classification scheme for atherosclerotic lesions. Arterioscler Thromb Vasc Biol 2000;20:126275.

3 Davies MJ, Richardson PD, Woolf N, et al. Risk of thrombosis in human atherosclerotic plaques: role of extracellular lipid, macrophage and smooth muscle cell content. Br Heart J 1993;69:377-81.

4 Casscells W, Hathorn B, David M, et al. Thermal detection of cellular infiltrates in living atherosclerotic plaques: possible implications for plaque rupture and thrombosis. Lancet 1996;347:1447-9.

5 Witztum TN. The oxidation hypothesis of atheroslerosis. Lancet 1994;344:793-5

6 Arbustini E, Morbini P, D'Armini AM, et al. Plaque composition in plexogenic and thromboembolic pulmonary hypertension: the critical role of thrombotic material in pultaceous core formation. Heart 2002;88: 177-82.

7 Egermayer P, Peacock AJ. Is pulmonary embolism a common cause of chronic pulmonary hypertension? Limitations of the embolic hypothesis. Eur Respir J 2000; 15:435-7.

8 Burke AP, Kolodgie FD, Farb A, et al. healed plaque ruptures and sudden coronary death: evidence that subclinical rupture has a role in plaque progression. Circulation 2001;103:934-40.

9 Beppu M, Hayashi T, Hasegawa T, et al. Recognition of sialosaccharide chains of glycophorin on damaged erythrocytes by macrophage scavenger receptors. Biochim Biophys Acta 1995;1268:9-19.

10 Antoku Y, Tsukamoto K, Miyoshi Y, et al. Correlations of elevated levels of hexacosanoate in erythrocyte membranes with risk factors for atherosclerosis. Atherosclerosis 2000;153:169-73.

11 Singh I, Khan M, Key L, et al. Lovastatin for X-linked adrenoleukodystrophiy. N Engl J Med 1998;339:702. 